\title{
El modelo de formación profesional en España
}

\author{
Ignasi Brunet, Rafael Böcker \\ Universitat Rovira i Virgili \\ ignasi.brunet@urv.cat; rafael.zavaro@urv.cat
}

Resumen: Este artículo expone como se ba ido configurando el modelo de formación profesional en España, al objeto de identificar cuál es el principal reto actual al que la formación profesional se enfrenta. Para ello bemos efectuado un diseño metodológico basado en las aportaciones de diversos autores a través del análisis e interpretación de textos. Los resultados han sido, identificar el motor de las distintas reformas de la formación profesional, y que tiene que ver con la necesidad de adaptar la formación profesional a los cambios en el entorno productivo. Esta necesidad de impulsar una mejor adecuación entre el sector de la formación profesional y el sistema productivo ha sido uno de los retos de la Ley Orgánica para la Mejora de la Calidad Educativa. Bajo el régimen de esta ley, España adopta, por un lado, un modelo de formación profesional en la línea del modelo dual, cuyo paradigma es Alemania. Por otro, esta ley bace bincapié en la prestación de servicios técnicos de los centros de formación profesional a las pequeñas empresas de su entorno, e impulsa la formación en competencias de innovación en los egresados de formación profesional.

Palabras clave: sistema educativo, innovación, formación profesional, reforma educativa, formación dual.

\section{The vocational education and training model in Spain}

Abstract: This article shows how it has been shaping the vocational education and training model in Spain in order to identify which is, currently, the main challenge that it faces. So we have made a methodological design based on the contributions from various authors through the analysis and interpretation of texts. The results have been, identifying the engine of the various reforms of the vocational education and training system, which has to do with the need to adapt vocational education and training to changes in the productive environment. This need to promote a better match between 
the vocational education and training sector and the productive system has been one of the challenges of the Organic Law for the Improvement of Educational Quality. Under the regime of this law, Spain adopts, on one hand, a model of vocational education and training in line with the dual model, whose paradigm is Germany. On the other hand, this law emphasizes the provision of technical services of vocational education and training centers to small businesses within their environment, and enhances skills training in innovation in vocational education and training graduates.

Keywords: educational system, innovation, vocational education and training, educational reform, dual training. 


\section{Introducción}

Según Wollschläger y Reuter-Kumpmann (2004) la revolución industrial provocó una reestructuración radical del sistema de «reabastecimiento de recursos humanos» en todos los países europeos y la emergencia de las clases sociales como nuevo modelo de estratificación social que acabará sustituyendo al estamental. Revolución que va a exigir al poder político que se haga cargo de la alfabetización de sus ciudadanos. Alfabetización que requería el nuevo orden político que surge en la modernidad, y que descansa sobre tres pilares: $a$ ) la consolidación del Estado-nación; conseguirlo es uno de los fines para cuyo logro fueron creados los sistemas educativos modernos nacionales; $b$ ) la emergencia de la burguesía como clase dominante que reclama el control político del Estado y trata de subordinarlo a sus intereses, enunciados en la ideología liberal o liberalismo, y c) el reconocimiento de los derechos de ciudadanía, que se irán ampliando, aunque muy lentamente, a toda la población (Archer, 1979). Pero, la alfabetización está también vinculada a la preocupación, fundamentalmente entre los patrones, por la formación profesional (FP) de los obreros. Preocupación que emerge a consecuencia de que las formas de aprendizaje tradicional vinculadas a las labores del campo o a la estructura de los gremios urbanos, dejaron de ser funcionales a finales del siglo xviII, como resultado de las nuevas necesidades de formación de la industria, orientada a implementar un ritmo y una disciplina fabril. Formación

que tenía dos dimensiones, a una la podemos llamar moral y a la otra técnica. La formación moral tenía que ver con la inculcación de los nuevos valores funcionales a la organización fabril del trabajo y en general con los valores emergentes del capitalismo. La formación técnica tenía que ver con las habilidades relacionadas con el manejo de la maquinaria utilizada en los distintos sectores fabriles (Martínez García y Merino, 2011: 14).

Este artículo expone como la preocupación técnica está detrás de las distintas reformas orientadas a adecuar el sector educativo de la FP a las necesidades técnicas del sistema productivo. Por ello, en el epígrafe segundo se detalla el proceso de constitución de los sistemas educativos nacionales, y que para el caso de España queda reflejado en la Ley Moyano de 1857. El epígrafe tercero desarrolla la crisis de la Ley Moyano que generó la industrialización tardía de España, y que llevó, en el marco del desarrollismo franquista, a la Ley General de Educación, de 1970. En el siguiente epígrafe se explica la reforma de la comprensividad que llevó a cabo en 1990, la Ley Orgánica General del Sistema Educativo (LOGSE). Esta ley fue contestada por sectores sociales y políticos conservadores, que realizaron una contrarreforma, la Ley Orgánica de Calidad de la Educación (LOCE), 
del 2001 y la LOMCE, de 2013. En esta última ley se recogen los requerimientos que el siglo xxi efectúa a la FP, y en el que la tecnología se ha convertido en un factor competitivo y en el que el trabajo tiene cada vez más un contenido cargado de tareas inteligentes que requieren creatividad e innovación, es decir, competencias en innovación. Finalmente, en las conclusiones destacamos que la opción por impulsar la creatividad y la innovación y por el modelo dual que efectúa la LOMCE responden al criterio «adecuacionista».

\section{El sistema educativo nacional español}

El desarrollo de la industrialización requirió de la constitución de un sistema educativo nacional (Archer, 1979), motivo por el que el Estado-nación asumió el control de la educación, dando lugar a los sistemas educativos nacionales. Sistemas que establecieron de forma obligatoria y suficiente una educación mínima para las masas populares, logrando extender la educación primaria a toda la población, colocando a la institución escolar en un terreno neutro, en el que se va a evaluar y clasificar a los ciudadanos, mediante pruebas objetivas por las que estos reciben una credencial que certifique sus talentos, sus méritos. Esta creencia está arraigada en la mayoría de los escritos de los reformadores educativos, y se halla en la base tanto de los proyectos liberales como de los socialdemócratas de educación. "Los objetivos que constantemente se han buscado desde el siglo XIX han sido la universalidad del sistema educativo - frente a la anterior escuela elitista - la igualdad de oportunidades para todos los niños — que se pensaba que podría lograrse mediante la estandarización del sistema escolar- y el individualismo, en dos sentidos: el sistema escolar trataría a los alumnos como individuos, no como miembros de colectividades previas, y habría de desarrollar el carácter de los alumnos como» personalidades únicas, es decir «desarrollar su ser moral e intelectual» (Martín Criado, 2003: 20). Esta dinámica la podemos observar en países como: Suecia (Boli, 1989), Francia, Gran Bretaña, Dinamarca o Rusia (Archer, 1979).

En el caso de España, esta dinámica quedó reflejada en la ley Moyano (1857), que subsistirá prácticamente hasta la Ley General de Educación (LGE) de 1970. Ley que consagró la división del sistema educativo en tres niveles, primario, medio y superior. Ley que declara la obligatoriedad de la enseñanza (de 6 a 9 años), si bien se olvidó de conceder su gratuidad (salvo para los pobres de solemnidad inscritos). Ley que prometió una escuela de niños y otra de niñas para cada quinientos habitantes, un instituto para cada provincia, y en fin, un total de 10 universidades. Ley que consagró «la separación de las enseñanzas medias entre la vía académica orientada a la universidad y la vía profesional con destino al mercado 
de trabajo, de ocupaciones de baja y media cualificación» (Martínez García y Merino, 2011: 14). De hecho, la ley Moyano lo que hizo fue intensificar el papel del Estado en materia de enseñanza, haciendo de la educación, sobre todo a nivel universitario, un asunto público. En ella se traza el camino para que la universidad y los licenciados salidos de ella, tengan un peso creciente en la vida pública española, concretamente al nivel de gestión del aparato estatal.

\section{Industrialización tardía y reformas educativas}

Según Merino (2005), la Ley Moyano entró en crisis porque la escuela tuvo que responder ante nuevas necesidades sociales (tanto de reproducción como de movilidad), lo que hizo necesario un nuevo marco normativo que consolidara de alguna manera el novedoso escenario social y económico que se estaba creando en la década de 1960, bajo el aperturismo de la dictadura franquista. En este escenario, cabe considerar que el cambio social en España ha sido cuando menos intenso, situación por otro lado típica en un país que ha sufrido una industrialización tardía, y que aporta

lo que Ishida denomina la ventaja del atraso, refiriéndose a que planificar las organizaciones e innovar tecnológicamente son procesos que consumen mucho tiempo y recursos. Los países que no se implican tempranamente en estos procesos de lenta transformación cuentan con una serie no desdeñables de beneficios, como la adopción de tecnologías que han sido creadas por otros previamente. Este proceso puede ser conducido desde arriba debido al escaso tejido empresarial de los países subdesarrollados (Marqués y Gil-Hernández, 2015: 63).

La LGE (1970) respondió a esta situación de industrialización tardía, presentando a la educación como la auténtica palanca para la movilidad social, la superación de las desigualdades y la consecución de una inserción profesional en los estratos altos o medios. Además, esta ley convirtió a la escuela básica en la llave para acceder a ulteriores oportunidades educativas. En este sentido, la Ley de 1970 supuso una mayor intervención formal del aparato escolar en el proceso de selección y legitimación social disfrazada de selección técnica. Para ello se impuso el principio neutral del mérito o del logro, y éste es precisamente el significado primitivo de la igualdad de oportunidades, y que explica el reforzamiento del aparato escolar que efectúa la ley de 1970 en tanto que instancia sancionadora del mérito escolar. Aparato que debe establecer la valía intelectual del alumno de modo neutro, atendiendo a su capacidad, a su talento, a su inteligencia, a sus aptitudes (Lerena, 1986). 
Debemos considerar, por otra parte, en el marco de la industrialización tardía y del desarrollismo franquista, que la LGE de 1970 fue la primera que se legisló en España como un "sistema educativo de masas". Una de cuyas contribuciones más relevante fue el que logró efectuar por primera vez en la historia de la educación española, la conexión entre la FP y el sistema educativo (Dávila et al. 2016). Sin embargo, se trató de una conexión peculiar, «en el sentido de lo que Baudelot y Establet (1976) llamaban la doble red: una red de escuela primaria y formación profesional corta de acceso a los oficios y ocupaciones de baja cualificación (PP) y una segunda de escolarización larga a través de la secundaria y la superior (SS). En el diseño de la LGE, paradójicamente, no estaba explicitado de esta manera, más bien al contrario», ya que «la idea original era establecer un tronco común y unificado con salidas profesionalizadoras al final de cada nivel». La formación profesional inicial (FP1) «era la salida para los que acababan la Educación General Básica (EGB)», la formación profesional media (FP2) «para los que acababan el bachillerato» y la formación profesional superior (FP3) «para los que acababan los primeros ciclos universitarios». De esta manera, la ley pretendía sustituir el modelo rígido de oficialía y maestría por un modelo en sintonía con las teorías meritocráticas del capital humano, con el objetivo de permitir la progresión de los individuos hasta el máximo de sus potencialidades, sus méritos y su valía personal» (Merino, 2005: 220). En este sentido, «la ley del setenta intenta configurar un subsistema de formación profesional basado sobre el papel en ciclos, es decir, en formaciones de corta duración (no más de dos años), de carácter 'vocacional'» (Merino, 2005: 220), y que tenga lugar

después de una etapa de formación general y con puentes de acceso al mundo del trabajo. El contexto político e ideológico no permitía romper la división entre trabajo manual e intelectual a edades tempranas (puesto que tras la EGB la única salida para el alumnado que no obtenía el graduado era la FP1) y adelantar en la línea de la comprensividad que los países anglosajones estaban promoviendo (con las comprehensive schools), e incluso en Alemania se hizo una tímida reforma comprensiva en medio de un sistema fuertemente dividido entre estudios académicos y profesionales (Merino, 2005: 221).

La LGE tuvo una contrarreforma con la promulgación de un decreto en el año 1974, que permitió de forma excepcional pasar de la FP1 a la FP2. Esta vía pasó de ser la excepcional a ser la más común (Planas, 1986). Además, en la práctica, la FP1 se convirtió en un recipiente de alumnos que habían fracasado en la enseñanza primaria y en el paso previo a la FP2, que inicialmente estaba prevista para bachilleres. De hecho, la FP1 tuvo una función más social que formativa o de preparación laboral, 
lo que en términos peyorativos se denominó el 'parking' escolar. El BUP (i.e. Bachillerato Unificado Polivalente) y el COU (i.e. Curso de Orientación Universitaria) se transformaron en un bachillerato de cuatro años con la vista puesta en la universidad. Aunque se introdujeron algunas materias de carácter tecnológico o aplicado, no pasaron de ser secundarias en el currículum y consideradas como 'marías. Con algunas excepciones, se consolidó el sistema de doble red o de doble vía tan bien analizados por Baudelot y Establet en la Francia de los años 70 del pasado siglo (Merino, 2013: 4-5).

Con esta reforma, la FP se convirtió «en un itinerario diferenciado y alternativo al tronco académico», asumiendo el rol «de referencia de prestigio y propedéutico» (Merino, 2005, 221).

\section{De la Ley Orgánica General del Sistema Educativo (1990)}

La promulgación de la LOGSE, en 1990, estuvo precedida por un ambiente de reforma en el que según García Lastra (2012) fueron planteadas tanto por la Ley de Reforma Universitaria de 1983, como por la de la secundaria y especialmente de la FP inicial o reglada, siguiendo unas finalidades generales bastante comunes en las reformas educativas europeas que se produjeron desde la década de 1980. Por un lado, aumentar la igualdad de oportunidades, disminuyendo la selección por origen social del acceso a los estudios medios y eliminando o disminuyendo el efecto de doble red (a partir de la doble titulación), que tiende a reproducir la clasificación entre trabajo manual e intelectual. Por otro, revalorizando la FP tanto en su imagen social como en la función de puente hacia el trabajo. Esta revalorización implicó aumentar los requisitos académicos para el acceso, así como estableció una metodología «adecuacionista» de las especialidades profesionales respecto a los sectores productivos y las ramas profesionales, las cuales se encontraban en profunda transformación con la aparición del capitalismo informacional. Además, esta revalorización estuvo pensada para desviar un porcentaje importante de alumnado de la vía académica universitaria.

Este nuevo planteamiento de la FP respondía a dos premisas fundamentales: 1) la extensión de la educación general era la mejor garantía para adaptarse a los cambios futuros. La especialización temprana o los cursos específicos para un empleo inmediato tenían el riesgo de preparar para un trabajo que pueda desaparecer en poco tiempo. La formación general debía combinar la formación académica con la formación más profesional. Esta FP, presente en la educación secundaria, recibe la denominación de FP de base; y 2) la FP específica, «tanto la 
de grado medio, que se cursa al término de la educación secundaria obligatoria, como la de grado superior, a la que se accede después del bachillerato, debe ser el puente entre la educación general y el mundo laboral» (Merino, 2005ः 225).

La LOGSE se presentó con la etiqueta de reforma comprensiva, ya que estuvo centrada en reducir la desigualdad basada en el origen social del alumnado, siguiendo con retraso la estela de las reformas educativas que se extendieron por Europa en la década de 1960. De hecho la incorporación a la escuela de los hijos de las clases trabajadoras, junto a la consideración de la ampliación de los años de escolarización obligatoria, se han de entender como una forma de evitar la segregación escolar temprana, al retrasar la elección de itinerarios escolares (o laborales), cuya meta era convertir a la escuela comprensiva en un baluarte fundamental para la consecución de la igualdad de oportunidades a partir de la segunda mitad del siglo xx (Puelles, 1991 y 2004).

«El núcleo duro de la comprensividad consistía en un currículum unificado, lo que» fue denominado como «tronco común, en la enseñanza secundaria inferior, de los 12 a los 16 años», así como «en la apuesta por las teorías constructivistas para trabajar la diversidad del alumnado en las aulas. Se pretendía, por lo tanto», retrasar la división curricular hasta los 16 años. «Para evitar la doble red y mejorar el prestigio de la formación profesional se tomaron dos medidas fundamentales: exigir el título de graduado en secundaria para los llamados ciclos formativos de grado medio (CFGM), es decir, igualar el requisito de acceso para la FP y el bachillerato; y exigir el título de bachiller para acceder a los llamados ciclos formativos de grado superior (CFGS), es decir, se desconectaban los dos niveles de formación profesional, los llamados ciclos formativos, que se definían como ciclos terminales o puente entre la formación y el mercado de trabajo» (Merino, 2013, 5). No obstante persistía un problema, el de qué hacer con los estudiantes que no obtenían el graduado en secundaria, y que al carecer del graduado de la ESO en la práctica les imposibilita insertarse en el mercado de trabajo. Ante este problema, «la LOGSE diseñó los llamados Programas de Garantía Social (PGS), que consistían en cursos de preparación profesional para oficios de poca cualificación», y cuya impartición «se externalizaban a agentes locales (municipalidades, fundaciones y entidades del Tercer Sector)» (Merino, 2013:5). Los PGS, de hecho, eran una reedición de la FP1 pero sin conexión a priori con los CFGM. Ideados desde una perspectiva asistencialista (el nombre ya es una muestra de esto) y diseñados desde la desregulación y la externalización del sistema educativo, constituyen el escalón más bajo de la FP, en este caso no reglada.

La LOGSE define una FP inicial o reglada (FPR), «basada en ciclos de duración corta al acabar los diferentes niveles de la formación general (ESO y ba- 
chillerato), como» «ya planteaba la LGE», al menos «sobre el papel». Además, «diferencia entre la FP de base y la FP específica [... ]. La primera estaría integrada en los ciclos obligatorios (primaria y ESO) y formaría parte del currículum general (con la introducción de la asignatura de tecnología en la ESO)» mientras que «la segunda serían ciclos formativos de grado medio y de grado superior, sin conexión ni pasarela», otorgando «una formación suficiente dentro de un campo profesional limitado, para encarar la transición a la vida activa»:

no sería coherente con este modelo conectar, a través de un curso puente, la FP de grado medio con la FP de grado superior. Se volvería al esquema de la LGE y conduciría a la pérdida de atractivo de la FP superior para aquellos alumnos que han cursado el bachillerato (Marchesi y Martín, 1998: 445).

«Más que volver al esquema de la LGE, se volvería así al esquema que supuso la contrarreforma de la LGE» (Merino, 2005: 225-226).

«Para evitar la doble vía, [...] se exige la misma titulación para hacer bachillerato y los CFGM» igualándolos en valor, al menos teóricamente. Esto se diseñó «para evitar la doble red y la segregación de la FP como vía para los no aptos desde el punto de vista académico. De alguna manera, los CFGM se hacen selectivos», pero no logra resolver «el problema de cómo formar a los no formados» (Merino, 2005: 226). «Se trata del dilema reflejado por De Pablo» (1997), en el sentido de que «si se aumenta el nivel de la FP, se hace más difícil para la población que tiene problemas con los estudios. Este dilema tiene, según este autor, dos salidas posibles: la primera es rebajar el nivel de la FP para que todo el mundo llegue. Esta salida generaría el siguiente círculo vicioso [... ]: desprestigio de la FP, poca valoración del mundo laboral, disminución de atractivo para los jóvenes... La segunda salida es mantener el nivel y ayudar a los alumnos con dificultades, por ejemplo, flexibilizando el currículum y permitiendo situaciones de estudios a tiempo parciales y/o alargando el horario de los ciclos para los alumnos con más dificultades» (Merino, 2005: 226).

Durante la década de 1990, y en el marco de la LOGSE, se aprobaron dos Planes Nacionales de FP $(1993,1998)$, que condujeron a la creación de la Ley Orgánica de Cualificaciones y Formación Profesional (Ley5/2002), que estableció que corresponde a la Administración General del Estado la regulación y la coordinación del Sistema Nacional de Cualificaciones y Formación Profesional (SNCFP), todo ello sin perjuicio de las competencias que correspondían a las Comunidades Autónomas y de la participación de los agentes sociales. Con posterioridad, debido a la grave crisis económica y financiera que se inició en 2007, el gobierno español diseñó una estrategia política a medio plazo basada en la sostenibilidad, que se ha concretado en la Ley de Economía Sostenible (LES) y 
en la Ley Orgánica Complementaria de la LES con la pretensión de reformar la economía española e impulsar la competitividad de las empresas españolas en los mercados internacionales (Marhuenda y Bernad, 2008). La LES (art. 1, ocho)

establece otra iniciativa para favorecer la incorporación al sistema de formación a través de la formación profesional a distancia. Mediante una plataforma de enseñanza a distancia extendida en todo el Estado, se podrán cursar módulos correspondientes a los títulos formativos de la formación profesional reglada y también se podrá cursar la 'formación complementaria' que se les exige a los trabajadores que, tras superar el proceso de evaluación y acreditación de las competencias profesionales adquiridas a lo largo de la vida laboral, quieren obtener un título de formación profesional reglada o el certificado de profesionalidad (Jiménez et alii 2013: 272-273).

\section{De la Ley Orgánica de Calidad de la Educación (2002) a la Ley Orgánica de Educación (2006)}

La LOGSE ha sido una reforma contestada por los sectores sociales y políticos conservadores, que criticaban el modelo «igualitarista» de la comprensividad, ya que en su opinión desincentivaba el mérito individual y hacía bajar el nivel educativo. Estos sectores en el período 1996-2004 se hicieron con el control del gobierno, y realizaron una contrarreforma que se denominó Ley Orgánica de Calidad de la Educación (LOCE, 2002), y estuvo auspiciada bajo el gobierno del Partido Popular (PP). Ley que no se pudo aplicar, ya que fue paralizada por el nuevo entorno a partir de marzo del 2004. Aun así, uno de los aspectos más relevantes de las leyes educativas del PP han sido los aspectos ideológicos, como su exaltación de la pedagogía del esfuerzo frente a la trasnochada pedagogía del interés, y la eliminación de mecanismos democráticos de elección de director. En la enseñanza secundaria obligatoria, esta ideología se reflejó mediante la instauración de los itinerarios, que no es más que una certificación de las prácticas de muchos centros de secundaria.

En el año 2006, con un nuevo gobierno socialdemócrata, se introdujo una nueva reforma, bajo la denominada Ley Orgánica de Educación (LOE). Ley que estructura la FP a partir de un conjunto de CFGM y GFGS «que tienen como finalidad preparar a los alumnos y alumnas para la actividad en un campo profesional y facilitar su adaptación a las modificaciones laborales que puedan producirse en su vida, así como contribuir a su desarrollo personal y al ejercicio de la ciudadanía democrática» (Gairín et alii 2009: 249). Además, la LOE incluyó como novedad en el currículo de la educación obligatoria, un número de com- 
petencias básicas que pasaron a constituir el núcleo curricular de la educación básica. La idea subyacente fue que la formación

no puede concebirse exclusiva ni principalmente como la adquisición de un conjunto de conocimientos distribuidos en diferentes ámbitos disciplinares, sino que debe tenerse también en cuenta la capacidad de utilizar los aprendizajes adquiridos para afrontar las situaciones nuevas que se plantean cotidianamente en la vida adulta (Tiana, 2011:64).

Este planteamiento se ajustó al proyecto DeSeCo (Definition and Selection of Competencies), que puso en marcha la OCDE, en 2002. El DeSeCo entendía la competencia como «la capacidad de responder a demandas complejas y llevar a cabo tareas diversas de forma adecuada» (BOE, 2015, 6986). Para ello identificó tres competencias clave: «actuar con autonomía, [... ] habilidad para utilizar de forma interactiva la lengua, [... ] el conocimiento [... ] y la tecnología, e interactuar en grupos heterogéneos" (Ramírez García et alii, 2015, 56). En el marco de este proyecto, las Recomendaciones del Parlamento Europeo y del Consejo Europeo (2006/962/CE) establecieron las siguientes competencias clave: «comunicación lingüística, competencia matemática y competencias básicas en ciencia y tecnología, competencia digital, aprender a aprender, competencias sociales y cívicas, sentido de la iniciativa y espíritu emprendedor, conciencia y expresión culturales»(BOE, 2015, 6988). Estas «Recomendaciones» se incluyeron en la LOE, que reorganizó

el segundo ciclo de la educación secundaria inferior, transformó los PGS en los Programas de Cualificación Profesional Inicial (PCPI), que introdujeron dos innovaciones muy significativas. La primera es que los chicos y chicas que hicieran estos programas podían obtener el graduado en secundaria y por lo tanto podría continuar en la enseñanza postobligatoria (Merino, 2013: 6).

La segunda es que se integraba en el sistema nacional de cualificaciones (en la línea del European Framework of Qualifications), es decir quedaba más integrado como el nivel 1 de formación profesional. Aunque no hay datos globales, sabemos por estudios sectoriales que ha aumentado el porcentaje de jóvenes que de los PCPI pasan a los CFGM (Merino, 2013: 6).

Con respecto a la FP, en 2005 (Real Decreto 1558/2005, de 23 de diciembre), se regulan los requisitos básicos de los Centros Integrados de FP (CIFP). Éstos pueden impartir formación reglada, ocupacional y continua. Los CIFP se plantearon como la gran apuesta para la integración entre la FP reglada o inicial y la formación para el empleo, y sobre todo para el desarrollo del conocimiento y la innovación en algunas familias profesionales. En 2006, se aprobó la Ley 2/2006, 
de 3 de mayo, que establecía las bases de la FP en el sistema educativo. En su artículo 30 se establecen los PCPI, y con el Real Decreto 229/2008, de 15 de febrero, se regulan los Centros de Referencia Nacional en el ámbito de la FP (CRNFP). Estos «son centros públicos cuya misión es realizar acciones de innovación y experimentación [...] en los diferentes sectores productivos y [...] referencia estatal en el ámbito de la familia profesional asignada» (BOE, 2008, 11069). En 2007, se regula el subsistema de FP para el empleo (Real Decreto 395/2007, de 23 de marzo). Este Real Decreto establece una reforma del modelo de FP en el ámbito laboral, incorporando mejoras en la adaptación de «la formación dirigida a trabajadores ocupados y desempleados a la nueva realidad económica y social» (BOE, 2015, 79781), y a las necesidades que demanda el mercado de trabajo.

En España, la formación de FP no reglada presenta algunas características que la alejan de los modelos internacionales, como el hecho incomprensible de que los dos principales componentes de la FP para el empleo, la ocupacional (o para desempleados) y la continua (o para trabajadores ocupados), nacieron independientemente. La primera, a partir de la entrada de España en la UE (1986); y la segunda, tras los acuerdos que a comienzos de la década de 1990 logran alcanzar patronal y sindicatos españoles sobre el destino de las cuotas para la formación de las cotizaciones sociales, que son asumidos por el gobierno central (AUTOR). A pesar de que con el Real Decreto 395/2007 se intentó integrar ambos sistemas (y superar así la anomalía que suponía el sistema español dentro del contexto europeo), la integración alcanzada es parcial. No está claramente delimitado o consensuado el papel de los diferentes gobiernos (central y autonómicos),

ni tampoco el de los agentes sociales y los centros de FP en todo el proceso. $\mathrm{Y}$ todavía se encuentra más lejana la integración de la FP para el empleo con la FP inicial, al persistir diferentes dependencias administrativas de una y otra, y no haberse desarrollado, tal como se preveían, los centros de FP integrales (Navarro, 2014: 55).

\section{La Ley Orgánica para la Mejora de la Calidad Educativa (2013)}

A finales de 2011, en un nuevo panorama socioeconómico marcado por la crisis financiera internacional, el PP recuperó el gobierno por mayoría absoluta, por lo que estuvo en condiciones de efectuar una nueva reforma educativa, tal y como sucedió en 2012 con el proyecto de la Ley Orgánica para la Mejora de la Calidad Educativa (LOMCE). Esta ley «propone una ruptura clara del modelo compren- 
sivo de la enseñanza secundaria obligatoria» (Merino, 2013: 11) y considera la FP como enseñanza aplicada; una enseñanza que ha de garantizar «que el alumnado adquiera y amplíe las competencias necesarias para su desarrollo profesional, personal y social» (Competencias, 2012).

«La ruptura del modelo comprensivo continúa con la rebaja a 15 años de la obligatoriedad, ya que tras cursar tercero, o incluso después de cursar segundo con un curso repetido», los estudiantes que no hayan superado «las competencias mínimas para seguir el currículum estándar podrán ser enviados a los llamados ciclos de Formación Profesional de Base» (Merino, 2013: 12). Por otro lado, «los programas de diversificación curricular, bautizados como programas de mejora del aprendizaje y el rendimiento, bajan de tercer a segundo curso, y se basarán en actividades prácticas. Finalmente, la ruptura del modelo comprensivo se completa con la propuesta de volver al doble título al finalizar la educación obligatoria, uno que habilite para las pruebas de acceso al bachillerato y otro que habilite para las pruebas de acceso a la formación profesional. La crítica al título único se salda con este retroceso histórico, sin tener en cuenta otras posibilidades apuntadas desde el mundo académico, como la supresión del título de graduado en educación secundaria o la vinculación del acceso al bachillerato o a la FP en función del rendimiento de determinadas materias durante la última etapa de la enseñanza obligatoria.

[...] En la propuesta de ley se refuerza la idea de la conectividad entre los diferentes ciclos de FP, e incluso entre la vía académica y la vía profesional, pero no se modifican los requisitos de acceso. Es decir, no se propone que haya una conexión directa entre los ciclos de FP de Base y los ciclos formativos de grado medio, ni entre éstos y los ciclos formativos de grado superior (Merino, 2013: 12).

Más específicamente, el sistema educativo español con la LOMCE queda del siguiente modo, «con anterioridad a los seis años, existe en España la Educación Infantil, que cubre la etapa de cero a seis años, pero la escolarización obligatoria comienza a los seis años, con la Educación Primaria, que dura seis años. A continuación, se cursa la Educación Secundaria Obligatoria durante cuatro años» (Pin et al, 2015: 5). Con la LOMCE, en la ESO se introducen «dos ciclos: el primer ciclo durará tres años y el segundo, un año, pudiendo los alumnos elegir en ese último año entre tres opciones diferentes: enseñanza académica, enseñanza aplicada o formación profesional básica [...]. En general, los alumnos suelen acabar esta etapa con dieciséis años y, a partir de ahí, pueden seguir en el sistema educativo con la Educación Secundaria postobligatoria o incorporarse al mercado laboral. Esta decisión es muy importante, ya que los estudiantes pueden elegir entre tres modalidades distintas de educación, de dos años cada una, o incorpo- 
rarse al mercado de trabajo: 1) Bachillerato; 2) Ciclo Formativo de Grado Medio; 3) Enseñanzas de Artes plásticas y Diseño, y Enseñanzas Deportivas de Grado Medio; 4) Mercado laboral» (Pin et alii, 2015:5).

«El Bachillerato suelen cursarlo aquellos alumnos que quieren acceder a los estudios universitarios», por lo que optan por cursar «y se considera el camino "académico" que prepara para una formación más intelectual. Al terminar el Bachillerato, sin embargo, también se puede optar por acceder al mercado laboral» (Pin et alii, 2015:5). Para matricularse en los CFGM es necesario haber obtenido el Graduado en la ESO. Estos ciclos «representan aproximadamente 2.000 horas de dedicación (dos años académicos) en distintos sectores de diferentes familias profesionales y permiten acceder directamente al mercado de trabajo con un título de técnico o continuar estudiando dos años más con un ciclo formativo de grado superior. Otro itinerario para acceder a los ciclos formativos de grado superior y a los estudios universitarios, es haber obtenido el Certificado de Bachillerato. Los candidatos tienen a su vez que haber cursado ciertas asignaturas relacionadas con el grado que piensan estudiar. Tras superar los ciclos formativos de grado superior, se obtiene el Diploma de Técnico Superior, y se puede proseguir con una formación de un grado relacionado con el campo de especialización. Tras esta formación, se puede acceder al mercado de trabajo o continuar los estudios en la Universidad. La formación profesional básica está diseñada específicamente para preparar a aquellos jóvenes que quieran acceder cuanto antes al mercado laboral» (Pin et alii, 2015:5).

«Existen también los [..+] PCPI para alumnos mayores de dieciséis años que no hayan obtenido el Graduado en la ESO, para facilitar su inserción laboral. Una vez superados los PCPI, los alumnos pueden continuar con estudios de FP de grado medio, que pueden durar uno o dos años, lo que da acceso directo al mercado de trabajo» (Pin et alii, 2015, 5); sin embargo, no ofrece la posibilidad directa de continuar estudiando en el sistema educativo. «La LOMCE prevé cambiar esto, de modo que el alumno que quiera continuar estudiando pueda acceder directamente a la FP de grado medio, así como a las otras posibles opciones. Existen otras alternativas en el sistema educativo español: son completamente vocacionales y se refieren a grados artísticos de música, arte y danza o deportes» (Pin et alii, 2015:5-6).

«Lo que pretende la LOMCE es que el segundo ciclo de la ESO [... ] sea un curso fundamental en el que los alumnos decidan si quieren continuar sus estudios por una vía académica (Bachillerato) o por una vía más aplicada (Ciclo Formativo de Grado Medio). De este modo, España seguiría un modelo en la línea del modelo alemán, cuyo nivel secundario básico oferta una educación más 
general, básica y práctica [...] o una educación enfocada a una vía más académica» (Pin et alii, 2015: 6). De hecho, el sistema dual de FP, cuyo paradigma es Alemania, Austria, Suiza y Dinamarca, es uno de los más valorados a nivel internacional, «debido a sus beneficios socioeconómicos, como bajas tasas de desempleo, mayor inserción del colectivo joven o una mejora de las competencias profesionales de la fuerza del trabajo. Se insiste así en la necesidad de exportar dicho modelo a otros países, ejemplo que estamos apreciando actualmente en España, con la [... L LOMCE» (Rego et alii, 2015: 150). La ley que regula este modelo fue "promulgada en 1968 y modificada en 2005, establece los derechos y obligaciones tanto de aprendices como de empleadores. Estipula así las normas relativas al período de aprendizaje del alumnado, así como objetivos, estructuración temporal y contenidos que deben examinarse» (Rego et alii, 2015: 151).

Una cuestión relativa a la FP que está muy presente en la actual Ley de educación española, la LOMCE, es aquella que recoge las conclusiones del Consejo Europeo de Bruselas de marzo de 2008. En estas conclusiones, señala Pedraza (2011), se destaca el potencial de innovación y la creatividad son un factor fundamental para el crecimiento, expresándose la necesidad de promover la creatividad y la innovación en la educación y la formación. Concretamente, se destacan la necesidad de: «a) Potenciar la creatividad y la capacidad de innovación en todos los niveles de la educación y la formación; $b$ ) Animar a los profesores a facilitar y fomentar entornos innovadores de aprendizaje; $c$ ) Facilitar y apoyar las redes y asociaciones entre la esfera educativa y el mundo laboral; d) Fomentar la creatividad y la innovación como objetivos de la cooperación europea, desarrollando entornos propicios para la creatividad y la innovación; y e) Fomentar el desarrollo, el intercambio y la difusión de buenas prácticas, utilizando los programas e instrumentos de la Unión Europea» (Pedraza, 2011: 2423). En este marco, la LOMCE presta atención a la necesidad de fomentar las capacidades creativas e innovadoras en los CIFP y en los CRNFP.

En este sentido, es importante resaltar la importancia de la formación en competencias de innovación en los centros de FP y de los servicios técnicos que dichos centros ofrecen a las pymes de su entorno. En cuanto a la innovación y a la posible contribución de los egresados a la transferencia de conocimiento o innovación, se ha encontrado que la Formación en Centros de Trabajo (FCT) contribuye de forma limitada a la transferencia de conocimiento vía el estudiante de FP, a excepción de aquellas empresas que, por su organización del trabajo y su concepción de los procesos de innovación, permiten una mayor participación de los alumnos en la innovación y en tareas de más responsabilidad (Brunet y Rodríguez-Soler, 2014). 
Se trata de empresas con sistemas de trabajo más colaborativos y modos de innovación más interactivos en donde se dan las condiciones para que los técnicos de FP participen más en procesos de innovación. En cuanto a los servicios de innovación, se ha encontrado que es una herramienta más útil que las FCT, y que la propia dinámica de los centros de FP permite una mejor adaptación a los ritmos y necesidades de las empreses, en espacial las pymes, que la que muestran otros agentes de I+D como las universidades. Una vez más, el tipo de empresa y las relaciones entre ambos actores marcan las pautas de colaboración entre ellos. De este modo, se observa una mayor relación entre centros de FP y empresas en aquellos territorios con un nivel elevado de cohesión y un nivel significativo de proximidad y conocimiento entre actores del territorio.

\section{Conclusiones}

La parte más novedosa de la LOMCE es la opción explícita por la formación dual. Formación que ofrece cualificaciones profesionales armonizando los procesos de enseñanza y aprendizaje entre instituciones formadoras y lugares de trabajo. Al respecto, señalar que en solo un año, la formación dual ha duplicado el número de estudiantes y empresas. Por ejemplo, en España, «en el año 2013 el $57 \%$ de la FP dual se dio en la modalidad en que la empresa y los centros son copartícipes en distinta proporción en los procesos de enseñanza y aprendizaje. La mayoría de los proyectos en 2013 se han aplicado a ciclos formativos de grado superior (72\%), frente al $28 \%$ de los de grado medio. Las familias profesionales relacionadas con el sector industrial suponen alrededor del 70\% de los proyectos abordados» (Pin et alii, 2015, 8). En cuanto al número de centros se ha dado «un incremento del 118\% con respecto a 2013 en el número de centros que ofertan ciclos formativos de FP en la modalidad dual. 375 centros van a desarrollar proyectos de FP dual en 2014, un 218\% más que en 2013. Se aprecian incrementos significativos sobre todo en Castilla-La Mancha, País Vasco, Cataluña y Comunidad Valenciana, respecto de 2013» (Pin et al, 2015, 9).

Con la opción por la formación dual, se trata de adecuar la FP con las necesidades técnicas del sistema productivo y poner punto y final a los siguientes estereotipos con respecto a la FP: «desfase recurrente entre necesidades de cualificación y oferta formativa, desconfianza respecto a la gente formada y falta de implicación en las empresas de los centros formativos» (Merino, 2005, 231). Estereotipos que han afectado y continúan afectando al debate en torno a la función social de la FP, desde la creación de la LGE, así como el hecho de que en la actualidad se le haya agregado la función económica de la FP y, concretamente, impulsar la creatividad de los egresados de FP y la prestación de servicios técnicos al 
tejido empresarial de su entorno, concretamente a las pymes. Esta función tiene que ver con la necesidad de promover las adaptaciones de los sistemas de FP a las necesidades actuales de cambio en el entorno productivo y al actual panorama laboral (Brunet et alii, 2017), así como incrementar el número de titulados de FP. 


\section{Bibliografía}

Archer, M. S. (1979). Social origins of educational systems, London, Sage.

Baudelot, C. y Establet, R. (1976). La escuela capitalista en Francia, Madrid, Siglo XXI.

BOE (2008, 25 de febrero) Real Decreto 229/2008, de 15 de febrero, por el que se regulan los Centros de Referencia Nacional en el ámbito de la formación profesional, Madrid, Ministerio de la Presidencia.

$\operatorname{BOE}(2015,29$ de enero). Orden ECD/65/2015, de 21 de enero, por la que se describen las relaciones entre las competencias, los contenidos y los criterios de evaluación de la educación primaria, la educación secundaria obligatoria y el bachillerato, Madrid, Ministerio de Educación, Cultura y Deporte.

$\operatorname{BOE}(2015,10$ de septiembre). Ley 30/2015, de 9 de septiembre, por la que se regula el Sistema de Formación Profesional para el empleo en el ámbito laboral, Madrid, Jefatura Del Estado.

Boli, J. (1989). New citizens for a new society. The institutional origins of mass schooling in Sweden, Oxford, Pergamon Press.

Brunet, I. y Rodríguez-Soler, J. (2014). «Formación profesional e innovación: estudio de la transferencia de innovación entre centros de FP y empresas», Revista de Educación, 365, pp. 177-201.

Brunet, I. (Coord.); Böcker, R.; Cascón, R.; Hernández, A. B.; Moral, D.; Papaoikonomou, E. (2017). La Formación Profesional e Innovación en Cataluña, Barcelona, Ediciones Bellaterra.

Competencias (2012, noviembre). «Futuro simple: 3.E. Un reto sin salida. El Título I. Las enseñanzas y su ordenación. La Formación profesional». Disponible en: <http://www.lascompetenciasbasicas.es/index.php/opinion/47articulos/205-futuro-simple-3e-un-reto-sin-salida.html>.

Dávila, P.; Murua, H. y Naya, L. M. (2016). «La Iglesia como agente promotor de la enseñanza profesional en el País Vasco y Navarra durante el franquismo», Revista española de pedagogía, 74:263, 167-185.

De PABlo, A. (1997). «La nueva formación profesional: dificultades de una construcción», Revista española de investigaciones sociológicas, 77-78, pp. 137-162.

Farriols, X.; Francí, J. y Inglés, M. (1994). La Formación Profesional en la LOGSE. De la ley a su implantación, Barcelona, Horsori-ICE de la UB.

Gairín, J.; Essomba i Gelabert, M. À.; Pozos, K. y Barrera, A. (2009). «La formación profesional y la formación del profesorado de FP en España: aproximaciones a la definición de cualificaciones y estándares», en GAIRÍN, J., Essomba i Gelabert, M. À. y Montané, D. (Coords.). La calidad de la for- 
mación profesional en Europa, hoy: análisis de la situación y propuestas de mejora (Barcelona, Wolters Kluwer España) pp. 243-262.

García Lastra, M. (2012). «Políticas y reformas educativas», en Trinidad, A.y Gómez, J. (Coord.). Sociedad, familia, educación (Madrid, Tecnos) pp. $103-115$.

Jiménez, M.; Luengo, J. y Sevilla, D. (2013). «Las políticas y legislación españolas de formación profesionalः las consecuencias de la aprobación de la Ley de Cualificaciones y de la Formación Profesional y de la Ley de Economía Sostenible», en Luzón, A. y Torres, M. (Comp.). Las politicas de formación profesional en España y en Europa: perspectivas comparadas (Barcelona, Octaedro) pp. 255-278.

Lerena, C. (1986). Escuela, ideología y clases sociales en España, Barcelona, Ariel. Marchesi, A. y Martín, E. (1998). Calidad de la enseñanza en tiempos de cambio, Madrid, Alianza.

Marhuenda, F. y Bernad, J. C. (2008). «El sistema de cualificación profesional en España y los/as trabajadores/as escasamente cualificados/as», Revista Europea de Formación Profesional, 42, pp. 91-101.

Marqués, I. y Gil-Hernández, C. J. (2015). «Origen social y sobreeducación en los universitarios españoles: ¿es meritocrático el acceso a la clase de servicio?», Revista española de investigaciones sociológicas, 150, pp. 89-112.

Martín Criado, E. (2003). «Una crítica de la sociología de la educación crítica», Anduli, 2, 9-27.

Martínez García, J. S. y Merino, R. (2011). «Formación profesional y desigualdad de oportunidades educativas por clase social y género», Revista Témpora, 14, pp. 13-37.

Merino, R. (2005). «Apuntes de historia de la Formación Profesional reglada en España: algunas reflexiones para la situación actual», Tempora, 8, pp. 211 236.

Merino, R. (2006)+ «¿Dos o tres itinerarios de formación profesional? Balance y situación actual en España», Revista europea de formación profesional, 37, pp. 55-69.

Merino, R. (2013). «Las sucesivas reformas de la formación profesional en España o la paradoja entre integración y segregación escolar», Education Policy Analysis Archives/Archivos Analiticos de Politicas Educativas, 21, pp. 1-15.

Navarro, M. (2014). El papel de los centros de formación profesional en los sistemas de innovación regionales y locales. La experiencia del País Vasco, Bilbao, Universidad de Deusto. 
OCDE (2002).Definition and Selection of Competencies: Theoretical and Conceptual Foundations (DeSeCo). Ver <http://www.oecd.org/edu/skills-beyondschool/definitionandselectionofcompetenciesdeseco.htm $>$ (Consultado el 02.IX.2016)

OCDE (2015). Politica educativa en perspectiva 2015: hacer posible las reformas. Ver <http://www.fundacionsantillana.com/upload/ficheros/noticias/201509/ politicas_educativas_para_la_web.pdf > (Consultado el 02.IX.2016)

Pedraza, B. (2011). «La formación profesional en Europa y en España: un nuevo contexto económico y social», EDUSK-Revista monográfica de educación Skepsis, 2:3, pp. 2407-2469.

Pin, J. R.; Roig, M.; Susaeta, L., y Apascaritei, P. (2015). La formación profesional dual como reto nacional, Pamplona, IESE Business School-Universidad de Navarra.

Planas, J. (1986). «La formación profesional en España: evolución y balance», Educación y Sociedad, 5, pp. 71-112.

Puelles, M. DE (1991). Educación e ideología en la España contemporánea, Barcelona, Labor.

Puelles, M. de (2004). Estado y educación en la España liberal (1809-1857). Un sistema educativo nacional frustrado, Barcelona, Pomares.

Ramírez García, A.; Renés, P. y Aguaded, I. (2015). «La competencia mediática en los criterios de evaluación del currículo de Educación Primaria», Aula Abierta, 44, pp. 55-62.

Rego, L*; Barreira, E. M. y Rial, A. F* (2015). «Formación profesional dual* comparativa entre el sistema alemán y el incipiente modelo español», Revista Española de Educación Comparada, 25, pp. 149-166.

Tiana, A. (2011). «Análisis de las competencias básicas como núcleo curricular en la educación obligatoria española», Bordón, 63:1, pp. 63-75.

Wollschläger, N. y Reuter-Kumpmann, H. (2004). «De la divergencia a la convergencia: Una historia de la formación profesional en Europa», Revista Europea de Formación Profesional, 32, pp. 6-17. 